\title{
Development and biodistrubition modeling of ${ }^{99 \mathrm{~m} T \mathrm{~T}-\mathrm{DTPA}}$
}

\author{
Mohammed Rabie Bricha', El-Mehdi Hamzaoui ${ }^{2}$, Kaddour Ben-Hamou ${ }^{1}$, Youssef Aboussaleh ${ }^{3}$, \\ Abdelhalim Mesfioui ${ }^{4}$, Abdellah Ettabia ${ }^{1}$, Hassan Moulahid ${ }^{1}$, Brahim Zoubir ${ }^{1}$, Ahmed El-Yahyaoui ${ }^{5}$ \\ ${ }^{I}$ Department of Radiopharmaceuticals Production, National Centre for Nuclear Energy, Sciences and Technologies, Rabat, Morocco \\ ${ }^{2}$ Department of IT Data Centre, National Centre for Nuclear Energy, Sciences and Technologies, Rabat, Morocco \\ ${ }^{3}$ Department of Compartmental Neurosciences and Nutritional Health Team, Ibn Tofail University, Faculty of Sciences, \\ Kenitra, Morocco \\ ${ }^{4}$ Department of Genitics - Neuroendocrinologiy and Biotechnology, Ibn Tofail University, Faculty of Sciences, Kenitra, Morocco \\ ${ }^{5}$ Department of Radiochemistry and Nuclear Chemistry, Mohammed V University, Faculty of Sciences, Rabat, Morocco
}

[Presented at the $4^{\text {th }}$ African Regional IRPA congress (AFRIRPA04), which was held from September 13-17, 2014 in Rabat, Morocco. This paper was reviewed and accepted by the scientific committee of the $4^{\text {th }}$ African Regional IRPA congress]

\section{Conference Proceeding}

\begin{abstract}
Purpose: In this study, the team modeled the biodistribution and the efficiency of two ${ }^{99 \mathrm{~m}}$-technetium diethylene triamine penta acetate ( $\left.{ }^{99 \mathrm{~m}} \mathrm{Tc}-\mathrm{DTPA}\right)$ based radiopharmaceuticals. Methods: The first radiopharmaceutical (DTPA-CNESTEN) is developed at the laboratories of the radiopharmaceutical production unit of the National Center for Nuclear Energy, Sciences and Technologies (CNESTEN-Morocco), and the second one is the commercial DTPA (DTPA-ref). Freeze-dried kits were successfully radiolabeled (radiochemical purity $>95 \%$ ) with the ${ }^{99 \mathrm{~m}}$ Tc. Then drugs were injected to male BALB/c mice. In each $2 \mathrm{~min}, 5 \mathrm{~min}, 15$ $\min , 1 \mathrm{~h}$ and $2 \mathrm{~h}$ time points after injections we evaluate tissue's distributions characteristics. At the end, an automatic modeling of the data were recorded from thyroid, blood and urinary excretion kinetics and biodistribution in mice using both DTPA kits. The study aimed to extract the parameters of the function used to fit the recorded data. Results and Conclusion: the team concluded that the biodistribution of ${ }^{99 \mathrm{~m} T c}$-DTPA can be modeled using a combination of two exponential parts. Moreover, the resultant plots showed that there is strong correlation between the formula found in literature and the one derived on the basis of the fit of data sets in this study. In addition, it was found that the biodistribution behaviors of the developed kit and the commercial one were very close. The obtained results suggest that the developed DTPA has practically the same kinetics as the commercial one.
\end{abstract}

Keywords: 99m-Technitium; Biodistribution; Data Fitting; DTPA; Modeling; Urinary Excretion Kinetics; CNESTEN

\section{Introduction}

Radiopharmaceuticals are radioactive compounds used for imaging and diagnosis of human diseases. Some of the radioactive are used for cancer therapy (about 5\%). ${ }^{1,2}$ A radiopharmaceutical has two components: a radionuclide and an agent (the pharmaceutical), which directs the radionuclide to a receptor antigen, ionic pump, or other site of interest within the body. Some radiopharmaceuticals are simple, such as the ionic form of the radionuclide, while most radiopharmaceuticals have a complex chemical structure where the radionuclide provides a signal, indicating the site of localization of the carrier molecule. ${ }^{2,3}$

Diethylene triamine penta acetic acid (DTPA) is one of pharmaceuticals. It used in different complexes for different objective, for examples: [111In-DTPA-DPhe1] octreotide is used for diagnostic stomastatin sintigraphy 4, 5, 6,7, Diethylene triamine penta acetic acid neolactosyl human serum albumin labeled with technetium- ${ }^{99 m}$ (99mTc-DTPA-LSA kit) afford the opportunity of hepatic receptor imaging. ${ }^{8}$ Diethylene triamine penta acetate galactosyl human serum albumin (GSA) is used as human hepatic asialoglycoprotein receptorbinding radiopharmaceutical in Japan since $1992 .{ }^{8}$ In constant, DTPA is an efficient candidate when it is radiolabeled with a therapeutic radioisotope such as ${ }^{177} \mathrm{Lu}$ or ${ }^{90} \mathrm{Y} .{ }^{9,}, 10,11,12$

Although this molecule has a negligible bridging capacity. ${ }^{13}$ It is used as a simple radiopharmaceutical (technetiated radiopharmaceutical). ${ }^{99 \mathrm{~m} T \mathrm{Tc}-\mathrm{DTPA}}$ is the most commonly used radiopharmaceutical for renography. ${ }^{14}$

The commercial DTPA kits are usually made of pentasodium or calcium trisodium salt of DTPA (diethylene triamine penta acetate. The freeze- dry kitcontains appropriate amounts of stannous chloride dehydrate in lyophilized form. Radio-

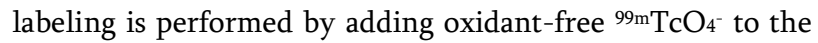
kit vial and mixing. In this study, attempt was made to fit the 
obtained experimental data in order to establish the developed DTPA biodistribution mathematical model. Also, the team had compared the behaviors of the developed kit (DTPA-CNESTEN) and the commercial one (DTPA-ref).

\section{Methods and Materials}

\section{Preparation of kit for ${ }^{99 \mathrm{~m} T c-D T P A}$}

\section{Reagents}

- Ethylenediamine pentaacetic acid (DTPA)

- Stannous chloride dihydrate: $\mathrm{SnCl}_{2} .2 \mathrm{H}_{2} 0$;

- Hydrochloric acid $\mathrm{HCl}$;

- Sodium hydroxide: $\mathrm{NaOH}$;

- Water for injection;

- Nitrogen gas.

\section{Technitium-99m}

Technetium-99m-pertechnitate was obtained from a commercial ${ }^{99 \mathrm{~m} M o /}{ }^{99 \mathrm{~m}} \mathrm{Tc}$ generator $(10 \mathrm{GBq})$ (schering/CIS- biointernational)

\section{Gamma counter}

The gamma counter used is NaI detector type COBRA 5002 from Packard.

\section{Radiolabeling}

Radiolabeling of DTPA-CNESTEN and the DTPA-ref, 1, were performed by reconstituting the freeze-dried kit using $5 \mathrm{~mL}$ of freshly eluted ${ }^{99} \mathrm{mcO}_{4}^{-}$solutions. The ${ }^{99 \mathrm{~m}^{-} \mathrm{TcO}_{4}-}$ solutions were eluted from ${ }^{99 \mathrm{~m}} \mathrm{Mo} /{ }^{99} \mathrm{~m} \mathrm{Tc}$ generator containing a maximum of $40 \mathrm{mCi}$ of activity. The ${ }^{99 \mathrm{~m} T c-D T P A}$ labeled in this manner should be stable for over 4 hours after labeling. ${ }^{15,16}$

\section{Animals}

To evaluate tissue's distributions characteristics of DTPACNESTEN and DTPA-ref, biodistribution studies were performed using male BALB/c mice, average weight about 50-60 g. The animals were carried from Pasteur Institute- Casablanca. During this study, they were reared in our laboratory and they had free access to food and water at all times. The room temperature was about $20^{\circ} \mathrm{C}$.

\section{Quality control}

\section{Radiochemical purity}

Radiochemical purity (RCP) is an important quality parameter for radiopharmaceuticals, as their radiochemical form determines their biodistribution. ${ }^{14,17}$ For this reason, the use of radiopharmaceutical in vivo needs RCP testing to be carried out just before administration to the patient. ${ }^{16,18,19}$

To determine radiochemical purity labeling efficiency of the product, European Pharmacopeia's chromatographic method, which is fast and easy, has been used. ${ }^{14}$ This method involves ascending instant thin layer chromatography (ITLC). The ITLC strips were utilized as the stationary phase and two different solvent systems as indexed in European Pharmacopeia 2008. The procedure involved spotting about $2 \mu \mathrm{l}$ sample of ${ }^{99 \mathrm{~m} T c}$ DTPA onto chromatography strips $10 \mathrm{~cm}$ in length. After developing in the solvent, the strips were cut into two portions and the activity in each portion was measured in the form of counts using a gamma counter. The percentage activity at the origin and the front were determined. The complex ${ }^{99 \mathrm{~m} T c}$ DTPA remained at the origin and free technetium traveled with the solvent (methyl-ethyl- ketone MEK) front $(\mathrm{Rf}=0.9-1.0)$. The percentage of colloid was determined using $0.9 \%$ solution of sodium chloride as the mobile phase (the ${ }^{99} \mathrm{~m}$ Tc-DTPA and $\mathrm{TcO}_{4}{ }^{-}$have about the same $\mathrm{Rf}$ in this system). Complexes were successfully labeled (RCP > 95\%) and stable about 24 hours.

\section{Biodistribution study}

Biodistribution studies regroup almost all of studies using radiopharmaceuticals. It is an important analytical step in preclinical studies. $^{20}$

After successful operations of radiolabeling, dilution (with $0.9 \% \mathrm{NaCl}$ solution) to obtain a volumetric activity about 400 $\mu \mathrm{Ci} / 5.4 \mathrm{~mL}$ and RCP was above 95\%. Mice were weighed and injected with $0.1 \mathrm{~mL}$ of the radiolabeled compound in the tail vein. After injections, the animals were kept in separately numbered beakers. And their urine was collected.

The injected activity is calculated by taking the difference between the weight of the syringe before and after the injection. At the end of $2 \mathrm{~min}, 5 \mathrm{~min}, 15 \mathrm{~min}, 1 \mathrm{~h}$ and $2 \mathrm{~h}$ time points, the animals were killed. They were dissected and blood sample was first taken by heart puncture. Then the other organs of interest were carefully dissected, placed in individual disposable plastic tubes and accurately weighed. The focus of this study was thyroid, blood, and urine. At least three animals were studied at each time point. After killing each mouse, the tail was removed and kept separately. The activity in the organs and the tail were measured in the gamma counter.

The total retained dose (\%TRD) estimated as follows:

$$
\% \operatorname{TRD}(\text { organ })=\frac{A}{B} \times 100
$$

Where, " $A$ " denotes the activity or counts in the organ of interest and " $\mathrm{B}$ " represents the activity or counts in all organs and the carcass except for the tail.

To accurately estimate the activity and to account for decay corrections in the ${ }^{99 \mathrm{~m} T c}$ activity, standard solutions of the radiopharmaceuticals were prepared. 


\section{Data Modeling}

Radiopharmaceuticals administered to humans disappear from the biological system through fecal or urinary excretion. This biological disappearance follows a bi-exponential law ${ }^{21}$, ${ }^{22}$, expressed as:

$$
y=a e^{b t}+c e^{d t}(e q .1)
$$

where, "t" designate time, "a" and "c" the intercepts and "b" and " $\mathrm{d}$ " are the slopes of the fast and the slow components of the plasma disappearance curve.

In this work, the team used the Open Curve Fitting Tool 3.3.1, incorporated under the MATLAB R2013b platform ${ }^{23}$, to compute the best values of the exponential parameters $a, b$, $c$ and $d$ with $95 \%$ confidence bounds. The optimal values of these parameters correspond to the minimum of the mean square error, which can be defined as the differences between the original data y (response value) and the predicted response value $\hat{y}$ at each predictor value.

For both sets of data, the mean values of the parameters defined above were calculated. The Table 1 below shows the obtained values of the model parameters; whereas Figure 1 illustrates the plot of the experimental data and the corresponding mathematical model.

TABLE 1: Parameter values of the bio-distribution data fitting model.

\begin{tabular}{lccccc}
\hline \hline Biological & \multicolumn{4}{c}{ Model Coefficients } & \\
\cline { 2 - 5 } parts & $\mathrm{a}$ & $\mathrm{b}$ & $\mathrm{c}$ & $\mathrm{d}$ & MSE \\
\hline Blood & 14.23 & -0.03257 & 0 & -0.03257 & 2.7603 \\
Urinary excretion & 57.08 & 0.004396 & -40.31 & -0.06128 & 5.9184 \\
Thyroid & 0.4199 & -0.01421 & 0 & -0.01421 & 0.1542 \\
\hline \hline
\end{tabular}

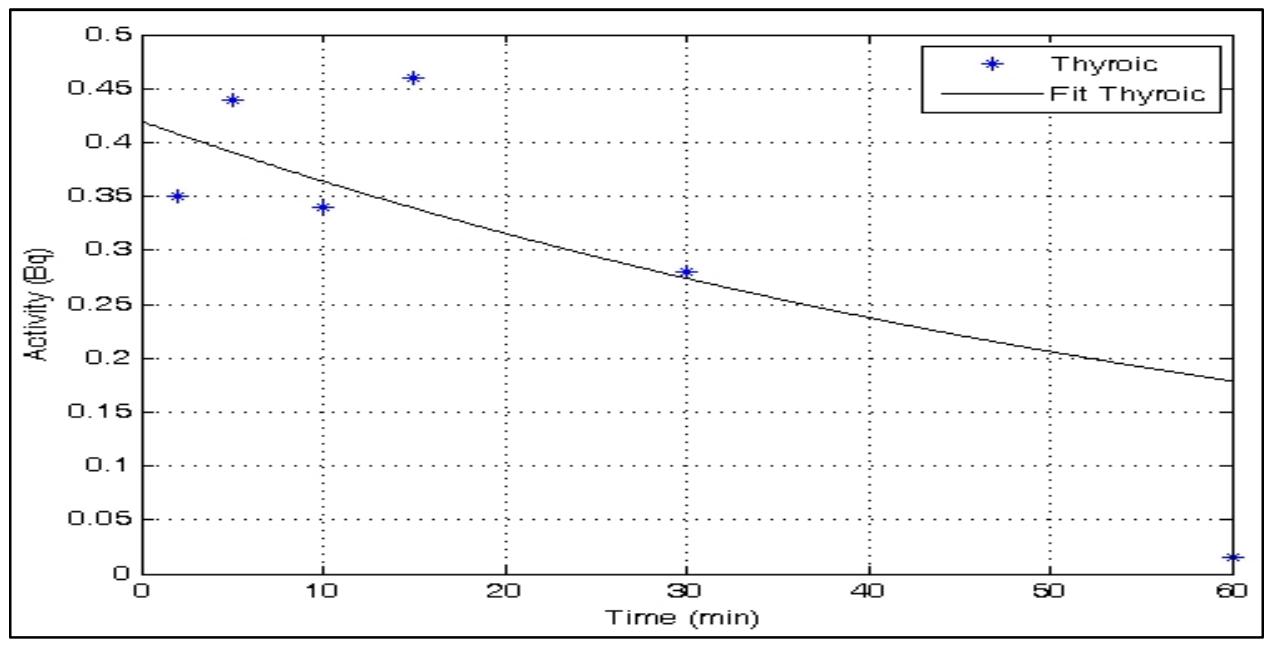

(a)

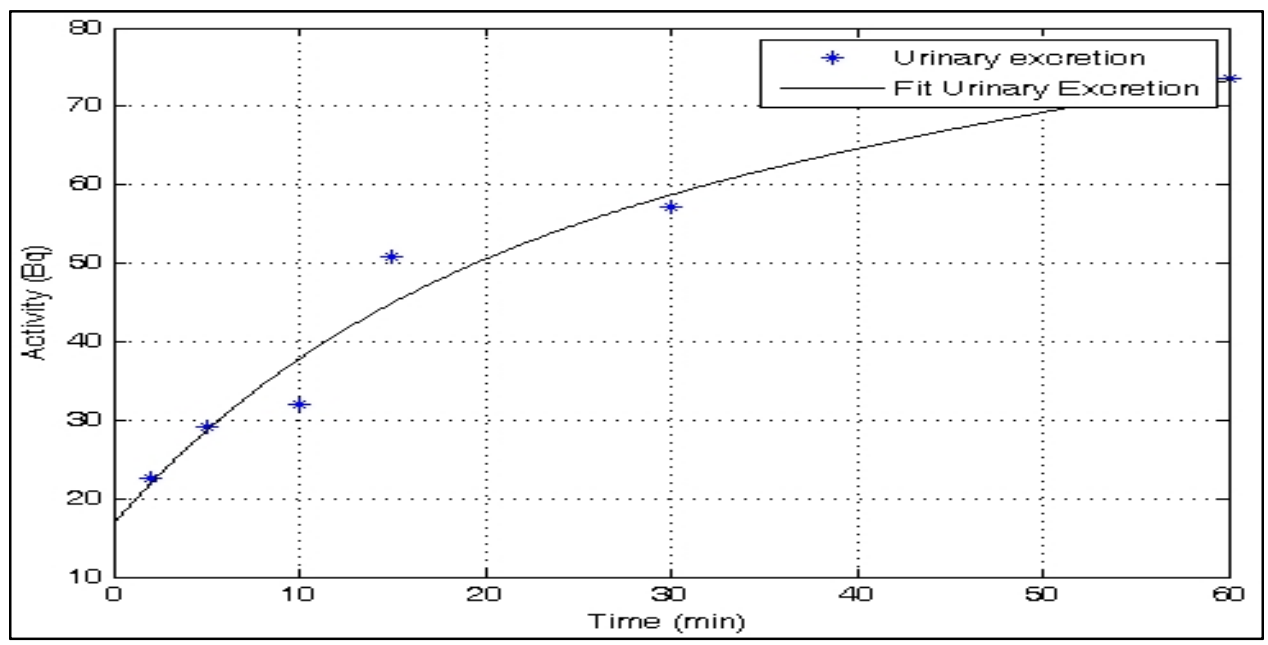

(b) 


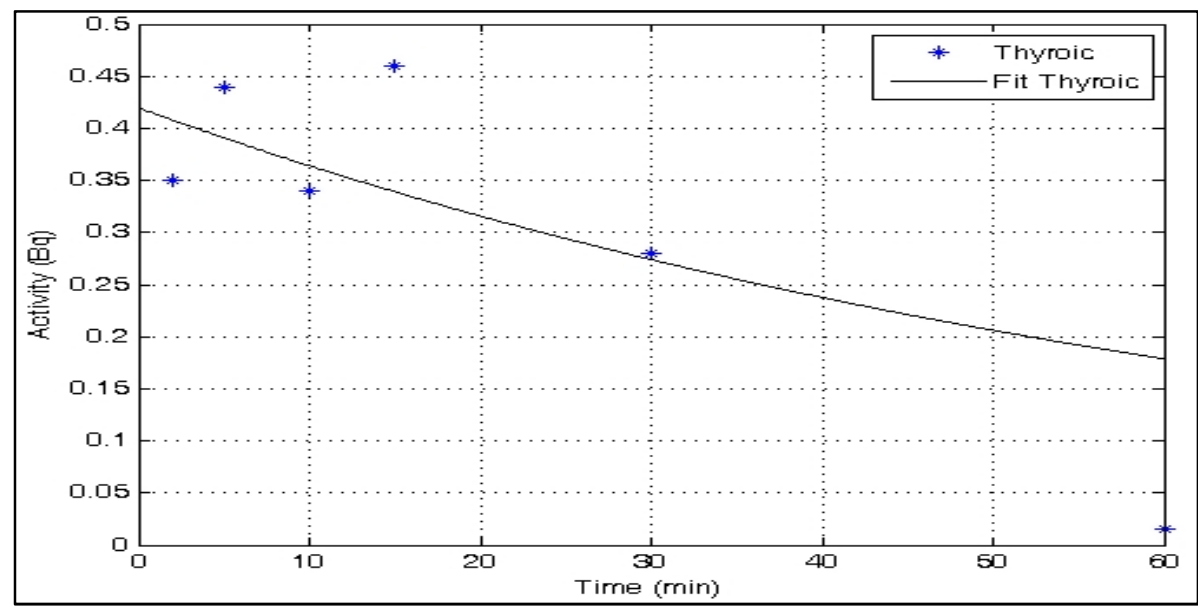

(c)

FIG. 1: Plot of the experimental data and the corresponding mathematical model in different biological parts. (a) Blood (b) Thyroid (c) Urinary excretion.

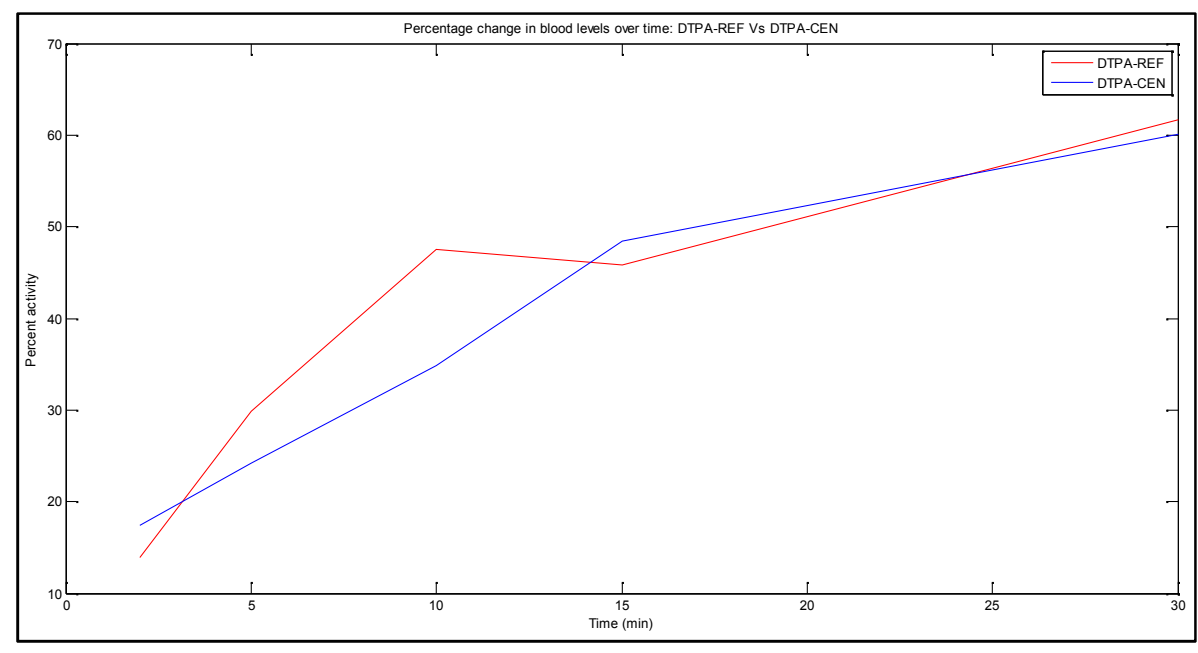

FIG. 2: Comparison of the developed DTPA and the commercial one in blood.

The comparison of the results obtained for the developed DTPA at the CNESTEN and the commercial one are illustrated in the Figure 2 below. This figure shows that the developed medicine has the same behavior as the commercial one.

\section{Conclusion}

In this study, the team concluded that the biodistribution of DTPA developed at the CNESTEN, can be modeled using a combination of two exponential parts. In addition, the graphics representations of the modeled data show that the drug evolution in both thyroid and blood has an opposite sense with its evolution in the urinary excretion. However, the obtained results proved that the developed DTPA has practically the same kinetics as the commercial one.

\section{Conflict of interest}

The authors declare that they have no conflicts of interest.
The authors alone are responsible for the content and writing of the paper.

\section{References}

1. Jongen Y. High beam intensities for cyclotron-based radioisotope production. International Atomic Energy Agency, Technical Document 1999;1065:133-8.

2. Saha GB. Fundamentals of Nuclear Pharmacy. $4^{\text {th }}$ ed, Springer, 1997.

3. Orsini F, Lorenzoni A, Erba PA, Masiani G. Radiopharmaceuticls for single-photoemission imaging and for therapy. Nuclear Oncology 2013: 21-34.

4. Robben J, Claude Reubi J, Pollak Y, Voorhout G. Biodistribution of [111In-DTPA-D-Phe1]- octreotide in dogs: uptake in the stomach and intestines but not in the spleen points towards interspecies differences. Nucl Med Biol. 2003;30:225-32. 
5. Bakker WH, Albert R, Bruns C, et al. [111InDTPA-D-Phe1]-octreotide, a potential radiopharmaceutical for imaging of somatostatin receptor-positive tumors: synthesis, radiolabeling and in vitro validation. Life Sci. 1991;49:1583-91.

6. Bakker WH, Krenning EP, Reubi JC, et al. In vivo application of [111In-DTPA-D-Phe1]-octreotide for detection of somatostatin receptor-positive tumors in rats. Life Sci. 1991;49:1593-601.

7. Krenning EP, Bakker WH, Kooij PP, et al. Somatostatin receptor scintigraphy with indium-111DTPA-D-Phe-1- octreotide in man: metabolism, dosimetry and comparison with iodine-123-Tyr3-octreotide. J Nucl Med. 1992;33:652-8.

8. Yang W, Zhao Z, Fang W, Zhang X. The preparation of 99mTc-DTPA-LSA and its instant lyophilized kit for hepatic receptor imaging. Appl Radiat Isot. 2013;74:1-5.

9. Lee SY, Hong YD, Kim HS, Choi SJ. Synthesis and application of a novel cysteine-based DTPA-NCS for targeted radioimmunotherapy. Nucl Med Biol. 2013;40:424-9.

10. Volkert WA, Hoffman TJ. Therapeutic radiopharmaceuticals. Chem Rev. 1999;99:2269-92.

11. Boswell CA, Brechbiel MW. Development of radioimmunotherapeutic and diagnostic antibodies: an inside-out view. Nucl Med Biol. 2007;34:757-78.

12. Anderson CJ, Welch MJ. Radiometal-labeled agents (non-technetium) for diagnostic imaging. Chem Rev. 1999;99:2219-34.

13. Husin H, leong YK, Liu JS. Surface force arising from adsorbed diethylenetriaminepentacetic acid (DTPA) and related compounds and their metal ions complexes in alumina suspensions. Colloids and Surfaces A: Physicochemical and Engineering Aspects 2013;422:172-80.
14. Borré MC, Tesán FC, Leonardi NM, et al. Vali ation of an alternative radiochemical purity method for [99mTc] pentetate ([99mTc]DTPA). Appl Radiat Isot. 2013;82:322-4.

15. European Pharmacopoeia, $6^{\text {th }}$ ed. Strasbourg, EDQM, 2008.

16. Tehnitium-99m Radiopharmaceuticals: Manufacture of kits. International Atomic Energy Agency, Technical Reports, STI/DOC/010/466, 2008.

17. Vallabhajosula S, Killeen RP, Osborne JR. Altered biodistribution of radiopharmaceuticals: role of radiochemical/pharmaceutical purity, physiological, and pharmacologic factors. Semin Nucl Med. 2010; 40:220-41.

18. World Health Organization. The WHO expert committee of specifications of pharmaceuticals preparations. Technical Report, 2008.

19. Maioli C, Bestetti A, Milani F, et al. Evaluation of different counting methods for use in radiochemical purity testing procedures for $99 \mathrm{mTc}$-labelled radiopharmaceuticals. Appl Radiat Isot. 2008; 66:556-9.

20. Pinto SR, Sarcinelle MA, de Souza Albernaz M, et al. In vivo studies: comparing the administration via and the impact on the biodistribution of radiopharmaceuticals. Nucl Med Biol. 2014;41:772-4.

21. Saha GB, Boyd CM. A two-compartmental model analysis of plasma clearance and urinary excretion data of 111In-DTPA in dogs. Int J Nucl Med Biol. 1982;9:122-5.

22. Saha GB, Boyd CM. Pharmacokinetic analysis of 99mTc-radiopharmaceutical data in humans by two-compartmental model. Int J Nucl Med Biol. 1982;9:126-8.

23. Mathworks Inc. The Open Curve Fitting Toolbox Software: User's Guide. 2013. www.mathworks.com 